\title{
The Weak and Main r-Process in Core-collapse Supernovae
}

\section{Shinya Wanajo*}

Department of Astronomy, School of Science, University of Tokyo, Bunkyo-ku, Tokyo, 113-8654

E-mail: wanajolastron.s.u-tokyo.ac.jp

\section{Yuhri Ishimaru}

Academic Support Center, Kogakuin University, Hachioji, Tokyo 192-0015

E-mail: kt13121@ns.kogakuin.ac.jp

\begin{abstract}
The astrophysical origin of the $r$-process nuclei is still unknown. Even the most promising scenario, the neutrino-driven winds from a nascent neutron star, encounters severe difficulties in obtaining requisite entropy and short dynamic timescale for the $r$-process. In this study, the effect of anisotropy in neutrino emission is examined with semi-analytic neutrino-driven wind models. It is shown that the neutrino heating rate from neutrino-antineutrino pair annihilation into electronpositron pairs can significantly enhance owing to the anisotropy and play a dominant role for the heating of wind material. A factor of five increase in the effective neutrino luminosity results in $50 \%$ higher entropy and a factor of ten shorter dynamic timescale owing to this enhanced neutrino heating. The nucleosynthesis calculations show that this brings the weak $r$-process model to the main $r$-process model, producing the third abundance peak $(A=195)$ and beyond.
\end{abstract}

International Symposium on Nuclear Astrophysics - Nuclei in the Cosmos - IX

June 25-30 2006

CERN, Geneva, Switzerland

\footnotetext{
*Speaker.
} 


\section{Introduction}

The astrophysical site of the rapid-neutron-capture nucleosynthesis ( $r$-process), which accounts for about half of nuclei heavier than iron, has been a long-standing mystery (see [1] for a recent review). During the last decade, the neutrino-heated ejecta from a nascent neutron star (neutrino-driven winds [2]) has been considered to be the most promising astrophysical site for the $r$-process. Previous studies show, however, severe problems in obtaining requisite high entropy and short dynamic timescale for the production of heavy $r$-process nuclei (e.g., [3, 4]). In this paper, it is shown that strong anisotropy in neutrino emission from the proto-neutron star, if it exists, naturally results in increasing entropy and reducing dynamic timescale of the winds. A more detailed discussion of the current results is presented in [5].

\section{Wind Models with Anisotropic Neutrino Emission}

The wind trajectories in this study are obtained using the semi-analytic, general relativistic model of neutrino-driven winds explored in [3, 4]. In this model, the system is treated as time stationary and spherically symmetric. The heating source that drives matter from the neutrino sphere is due to neutrino interactions. Heating is due to $v_{e}$ and $\bar{v}_{e}$ capture on free nucleons $\left(\dot{q}_{v N}\right)$, neutrino scattering by electrons and positrons $\left(\dot{q}_{v e}\right)$, and neutrino-antineutrino pair annihilation into electron-positron pairs $\left(\dot{q}_{v v}\right)$. Cooling is due to electron and positron capture on free nucleons $\left(\dot{q}_{e N}\right)$ and electron-positron pair annihilation into neutrino-antineutrino pairs $\left(\dot{q}_{e e}\right)$.

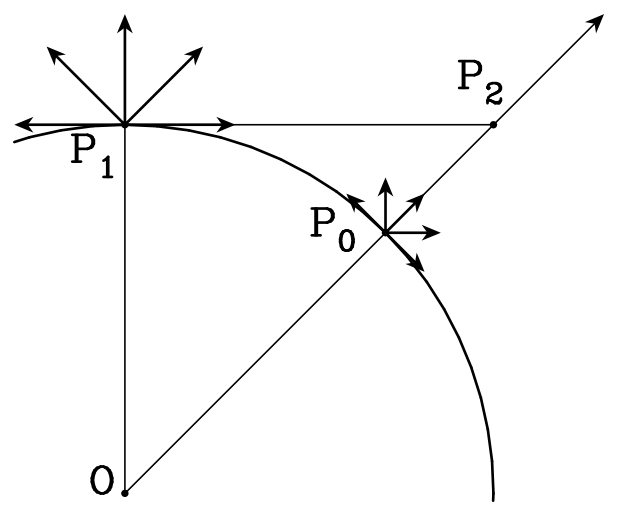

Figure 1: Illustration of asymmetric neutrino emission. $\mathrm{O}$ is the center of the neutron star. Strong neutrino emission from the "hot spot" near the point $\mathrm{P}_{1}$ on the neutrino sphere is assumed, otherwise being isotropic. A wind blowing from a nearby point $\mathrm{P}_{0}$ with the (weaker) isotropic neutrino emission $\left(L_{v}\right)$ suddenly see a larger number of neutrinos $\left(L_{v 2}\right)$ when passing $\mathrm{P}_{2}$.

The mass and radius of the neutron star are taken to be $M=1.4 M_{\odot}$ and $R=10 \mathrm{~km}$, respectively. The neutrino luminosity of one specific flavor is assumed to be the same for all other flavors, which is taken to be a constant value $L_{v}=1 \times 10^{51} \mathrm{ergs} \mathrm{s}^{-1}$. As explored in previous studies, this typical choice of parameter set (with isotropic neutrino emission) results in the production of weak $r$-process nuclei (up to $A \sim 130$ ) [3].

In this study, anisotropy in neutrino emission is modeled schematically as follows. Given there is substantially higher neutrino emission from the "hot spot", which is marked by the point 
$\mathrm{P}_{1}\left(\mathrm{OP}_{1}=R\right)$ in Figure 1. At the point $\mathrm{P}_{0}\left(\mathrm{OP}_{0}=R\right)$ nearby $\mathrm{P}_{1}$, the ejection of matter is due to the local (lower) isotropic neutrino emission around $\mathrm{P}_{0}$. The matter suddenly sees a substantially larger number of neutrinos when passing through the point $\mathrm{P}_{2}$. This sudden increase of the neutrino number density at $\mathrm{P}_{2}$ is approximated by a jump of the neutrino luminosity from the original value $L_{v}=1 \times 10^{51} \mathrm{ergs} \mathrm{s}^{-1}$ for $R<r<R_{2}$ to the effective luminosity $L_{v 2}$ for $r \geq R_{2}$, where $r$ is the distance from the center $\mathrm{O}$ and $R_{2}=\mathrm{OP}_{2}$.

\begin{tabular}{cccccccccc}
\hline \hline & $\begin{array}{c}R_{2} \\
\text { Model }\end{array}$ & $\begin{array}{c}L_{v 2}\left(10^{51}\right. \\
\mathrm{km})\end{array}$ & $\begin{array}{c}\dot{M}\left(10^{-6}\right. \\
\left.\mathrm{rgs} \mathrm{s}^{-1}\right)\end{array}$ & $\begin{array}{c}\left.M_{\odot} \mathrm{s}^{-1}\right) \\
\left.\mathrm{g} \mathrm{cm}^{-3}\right)\end{array}$ & $\begin{array}{c}T_{13}\left(10^{10}\right. \\
\mathrm{K})\end{array}$ & $\begin{array}{c}s \\
\left(N_{A} k\right)\end{array}$ & $\begin{array}{c}\tau_{\mathrm{dyn}} \\
(\mathrm{ms})\end{array}$ & $Y_{n} / Y_{h}$ & $\left\langle A_{h}\right\rangle$ \\
\hline $\mathrm{A} 1$ & 10 & 1.0 & 3.86 & 3.49 & 2.06 & 117 & 14.1 & 6.01 & 107 \\
$\mathrm{~A} 2$ & 10 & 2.0 & 13.3 & 5.98 & 2.35 & 103 & 7.20 & 8.16 & 109 \\
$\mathrm{~A} 3$ & 10 & 3.0 & 27.4 & 8.10 & 2.54 & 95.5 & 4.99 & 6.02 & 107 \\
$\mathrm{~A} 4$ & 10 & 4.0 & 46.0 & 9.75 & 2.66 & 90.7 & 3.90 & 6.02 & 107 \\
$\mathrm{~A} 5$ & 10 & 5.0 & 68.7 & 11.9 & 2.80 & 87.0 & 3.26 & 10.3 & 111 \\
$\mathrm{~B} 2$ & 12 & 2.0 & 4.88 & 2.68 & 1.88 & 131 & 7.45 & 18.0 & 118 \\
$\mathrm{~B} 3$ & 12 & 3.0 & 5.84 & 2.08 & 1.73 & 145 & 4.44 & 38.9 & 135 \\
$\mathrm{~B} 4$ & 12 & 4.0 & 6.84 & 1.65 & 1.61 & 161 & 2.72 & 80.7 & 170 \\
$\mathrm{~B} 5$ & 12 & 5.0 & 7.93 & 1.15 & 1.46 & 180 & 1.65 & 176 & 230 \\
$\mathrm{C} 2$ & 15 & 2.0 & 4.15 & 3.37 & 1.99 & 127 & 9.76 & 12.1 & 113 \\
$\mathrm{C} 3$ & 15 & 3.0 & 4.41 & 3.25 & 1.94 & 136 & 7.08 & 20.9 & 120 \\
$\mathrm{C} 4$ & 15 & 4.0 & 4.68 & 3.25 & 1.90 & 147 & 5.15 & 34.7 & 131 \\
$\mathrm{C} 5$ & 15 & 5.0 & 4.97 & 3.10 & 1.84 & 159 & 3.69 & 58.3 & 153 \\
D2 & 20 & 2.0 & 3.93 & 3.45 & 2.04 & 122 & 11.9 & 8.43 & 109 \\
D3 & 20 & 3.0 & 4.00 & 3.53 & 2.04 & 127 & 10.2 & 11.3 & 112 \\
D4 & 20 & 4.0 & 4.08 & 3.50 & 2.02 & 132 & 8.69 & 14.9 & 115 \\
D5 & 20 & 5.0 & 4.15 & 3.48 & 2.01 & 137 & 7.43 & 19.3 & 119 \\
\hline
\end{tabular}

The wind models considered in this study are listed in the first column of Table 1, where $R_{2}$ (second column) and $L_{v 2}$ (third column) are taken to be 10, 12, 15, and 20 in units of km, and 1.0, 2.0, 3.0, 4.0, and 5.0 in units of $10^{51} \mathrm{ergs} \mathrm{s}^{-1}$. The resulting net heating rate ( $\dot{q}$; top panel) and each heating rate ( $\dot{q}_{v N}, \dot{q}_{v e}$, or $\dot{q}_{v v} ;$ bottom panel) for models A1, A5, B5, C5, and D5 are shown in Figure 2, as functions of $r$. Note that A1-A5 are isotropic wind models (i.e., $R_{2}=R$ ).

For isotropic winds (A1-A5 in Table 1), the higher $L_{v 2}\left(=L_{v}\right.$ in these cases) results in shorter dynamic timescale $\tau_{\text {dyn }}\left(\equiv|\rho /(d \rho / d t)|_{T=0.5 \mathrm{Mev}}\right)$ and lower asymptotic (i.e., maximum) entropy $s$. In contrast, for anisotropic models, an increase of $L_{v 2}$ (for $r \geq R_{2}$ ) is quite efficient both to increase entropy and to reduce dynamic timescale (Table 1). The reason is that the matter has been already lifted with low $L_{v}\left(=1 \times 10^{51} \mathrm{ergss}^{-1}<L_{v 2}\right)$ and thus with small $\dot{M}$. Therefore, the density (and temperature) at arbitrary $r$ is significantly small compared to the corresponding isotropic wind. This can be seen in the 5th (and 6th) column in Table 1, which lists the density $\rho_{13}$ (and temperature $T_{13}$ ) at $r=13 \mathrm{~km}$ (see about one order difference in $\rho_{13}$ for A5 and B5).

For isotropic wind models, the five times greater neutrino luminosity simply results in the increase of $\dot{q}$ with the same factor (A1 and A5 in Fig. 2, top panel). This does not hold, however, for anisotropic wind models. For model B5, the maximum $\dot{q}$ is as twice large as that for model 


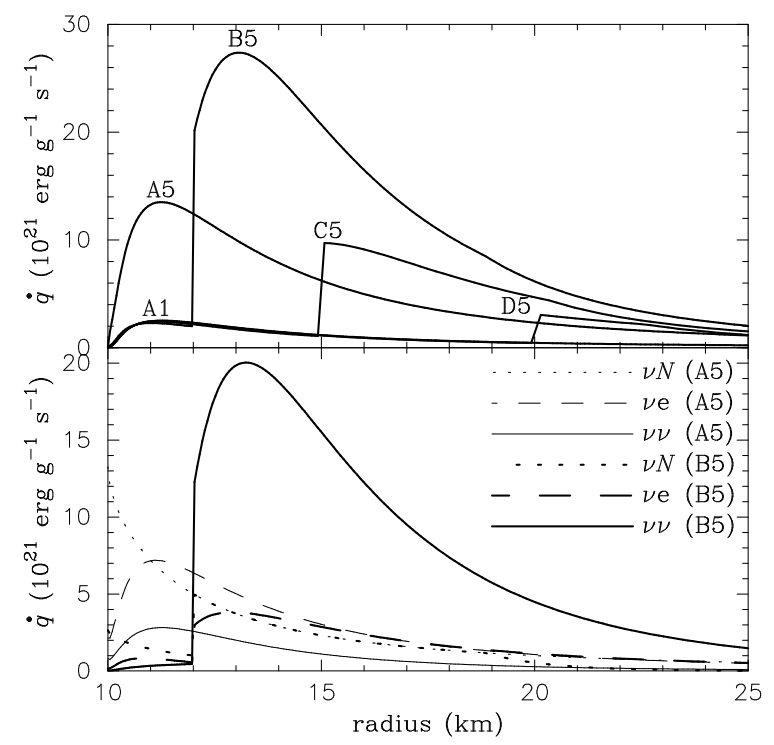

Figure 2: Top: Net neutrino heating rates for wind models A1, A5, B5, C5, and D5 listed in Table 1, as a function of $r$. Jump of the heating rate at $r=R_{2}$ is due to the sudden increase of effective neutrino luminosity from $L_{v}$ to $L_{v 2}$. Bottom: Heating rates $\dot{q}_{v N}, \dot{q}_{n u e}$, and $\dot{q}_{v v}$ as functions of $r$. Thin and thick lines are for wind models A5 and B5 (listed in Table 1), respectively.

A5 (with the same $L_{v 2}$ ), and more than 10 times larger than that for model A1 (with the same $L_{v}$ ). This can be explained as follows. As shown in Figure 2 (bottom panel), for isotropic winds (thin lines; A5), the heating is mainly due to $\dot{q}_{v N}$ and $\dot{q}_{v e}$, while $\dot{q}_{v v}$ plays only a minor role (cooling terms $\dot{q}_{e N}$ and $\dot{q}_{e e}$ are not shown). In contrast, for anisotropic winds (thick lines), the neutrino pair annihilation $\dot{q}_{v v}$ plays a crucial role, whose peak (in B5; at $r \approx 13 \mathrm{~km}$ ) is a factor of seven higher than that in A5.

\section{Nucleosynthesis in Winds}

Adopting the wind trajectories discussed in $\S 2$ for the physical conditions, the nucleosynthetic yields are obtained by solving an extensive nuclear reaction network. The network consists of 6300 species between the proton and neutron drip lines (for more detail, see [6]). Each calculation is initiated when the temperature decreases to $T_{9}=9$ (where $T_{9} \equiv T / 10^{9} \mathrm{~K}$ ). The initial compositions are given by $X_{n}=1-Y_{e}$ and $X_{p}=Y_{e}$, respectively, where $X_{n}$ and $X_{p}$ are the mass fractions of neutrons and protons, and $Y_{e}$ is the initial electron fraction (number of proton per nucleon) at $T_{9}=9$. In this study, $Y_{e}$ is taken to be 0.4 .

The nucleosynthesis results for models listed in Table 1 (except for A1-A5) are shown in Figure 3, as a function of atomic mass number. For anisotropic wind models with $R_{2}=12 \mathrm{~km}$ (B2-B5), the effect of anisotropic neutrino emission is evident. A factor of three or four increase in $L_{v 2}$ (B3 and B4 in Table 1) leads to $s \approx 150-160 N_{A} k$ and $\tau_{\mathrm{dyn}} \approx 3-4 \mathrm{~ms}$, resulting in the $r$-process nucleosynthesis (Fig. 3). For model B5, the high entropy $\left(=180 N_{A} k\right)$ and short dynamic timescale $(=1.65 \mathrm{~ms})$ of the wind drive the nuclear matter to the actinide region. The neutron-toseed abundance ratio at the beginning of the $r$-process, defined as $T_{9}=2.5$, is $Y_{n} / Y_{h}=176$ and the 


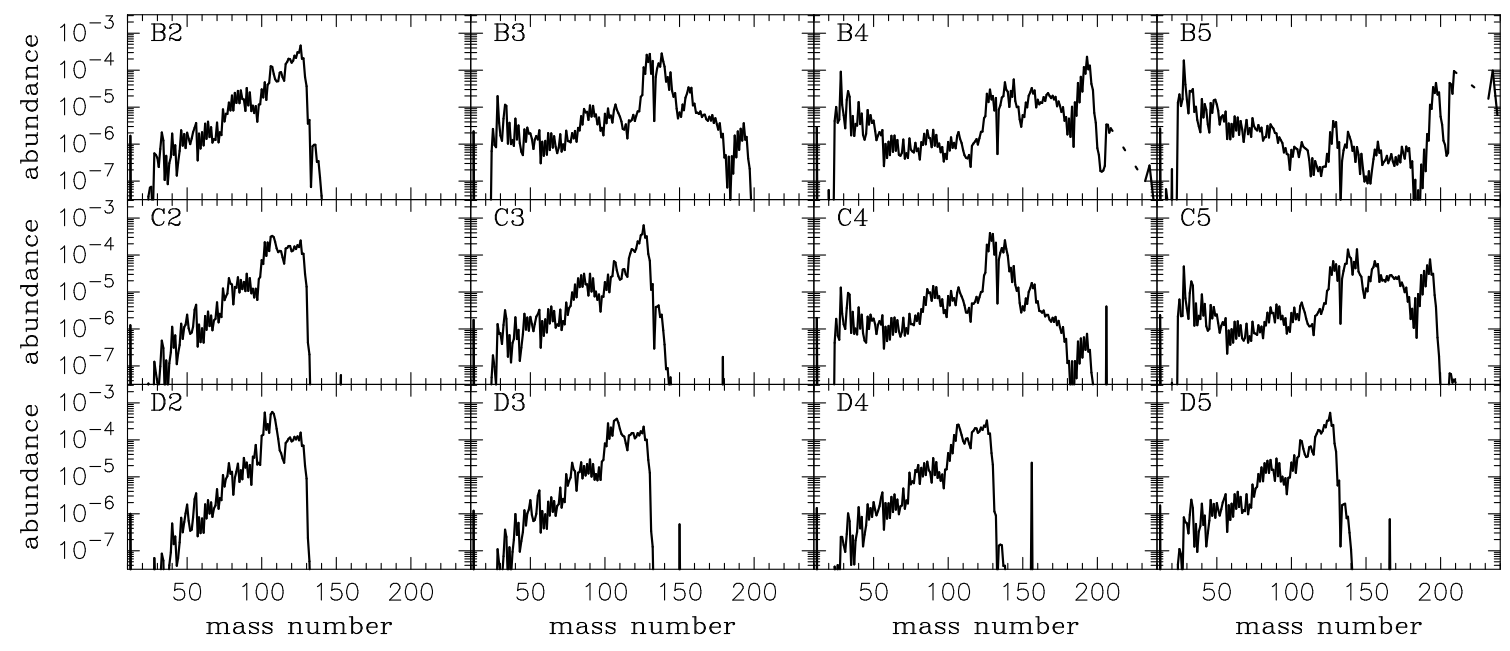

Figure 3: Final abundances obtained by the nucleosynthesis calculations for wind models listed in Table 1 (except for A1-A5) as a function of atomic mass number.

final averaged mass number of heavy nuclei with $Z>2$ is $\left\langle A_{h}\right\rangle=230$ (Table 1). For the models with $R_{2}=15 \mathrm{~km}$ (C2-C5), the $r$-process still takes place when $L_{v 2}$ is four or five times higher than $L_{v}$ (models C4 and C5). For $R_{2}=20 \mathrm{~km}$ (D2-D5), the effect of anisotropic neutrino emission is not important and the nucleosynthesis results are not significantly different from the isotropic cases (A1-A5).

\section{Implications}

In this study, the effects of anisotropy in neutrino emission for the $r$-process nucleosynthesis in proto-neutron-star winds were examined, using the spherically symmetric, steady outflow model of neutrino-driven winds. It was shown that strong anisotropy, if it exists, can be an additional energy source [7] to heat the wind material. A factor of four or five enhancement in effective neutrino luminosity results in the significant increase of entropy and shortening of dynamic timescale of outgoing neutrino-heated ejecta. This is mainly due to the boosted neutrino heating from annihilation of neutrino-antineutrino pairs into electron-positron pairs as a result of anisotropic neutrino emission. This provides the physical condition suitable for the robust $r$-process, producing the third abundance peak $(A=195)$ and beyond (see [5] for more detail).

It is conceivable that asymmetric neutrino emission can be associated with the anisotropic matter distribution near the neutrino sphere. As an example, [8] suggested that the non-spherical neutrino sphere owing to rapid rotation leads to anisotropic neutrino heating with the pole-to-equator ratio of a few to more than 10 . This may result in strong contrast in neutrino emission on the neutrino sphere, which forms an effective "hot spot" around the rotational axis. A recent work with more sophisticated neutrino-transport scheme by [9] showed, however, that the pole-to-equator flux ratio is at most a factor of two, even for a rather rapidly rotating core. This is a consequence that the radiation field is smoothened by the many neutrino sources above the neutrino sphere (e.g., convective bubbles) at the early phase ( $<1 \mathrm{~s}$ after core bounce). Nevertheless, all the convective 
bubbles are evacuated during the late wind phase $(\sim 10 \mathrm{~s})$ and a strong contrast of neutrino flux might form on the neutrino sphere for a rapidly rotating core.

Another possibility of anisotropic neutrino emission might be due a global fluid instabilities of neutrino-heated matter as observed in multi-dimensional hydrodynamic simulations. Recent works have shown that hydrodynamic instabilities can lead to low-mode ( $l=1$ in terms of an expansion in spherical harmonics of order $l$ ) oscillation of the convective fluid flow in the neutrino-heated layer behind the shock [10]. The presence of such a low convective mode results in the pair of a single outflow and a narrow accretion flow that creates the "hot spot" on the neutron star surface. It should be noted, however, that the two-dimensional simulations by [10] showed that the anisotropy of the accretion luminosity owing to this flow appears to be only a few percent (at least during the early phase up to $\sim 1 \mathrm{~s}$ after core bounce). A future investigation relevant for the wind phase $(\sim 1-10 \mathrm{~s})$ will be needed to examine the degree of anisotropic neutrino emission from such an accretion flow.

Given that one of the above (or another unknown) mechanism works, a constraint for the $r$ process may be obtained from the condition that creates the "hot spot" owing to, e.g., rapid rotation or long lasting accretion flow. It is conceivable that only a limited fraction of supernovae create the "host spot" relevant for the current scenario (e.g., rapid rotators or less-energetic supernovae that form the long lasting accretion flow). This can be a reasonable explanation for that the spectroscopic analysis of extremely metal-poor stars and Galactic chemical evolution study imply only a limited fraction of core-collapse supernovae undergo the $r$-process nucleosynthesis [11, 12].

The implications in this study must be tested by future multi-dimensional simulations of corecollapse supernovae for long duration $(\sim 10 \mathrm{~s})$ with accurate neutrino transport. Systematic calculations of nucleosynthesis with such hydrodynamic trajectories will be also needed to investigate the contribution to the Galactic chemical evolution of $r$-process nuclei.

\section{References}

[1] S. Wanajo and I. Ishimaru, Nucl. Phys. A, 777, 676 (2006).

[2] S. E. Woosley, J. R. Wilson, G. J. Mathews, R. D. Hoffman, and B. S. Meyer, Astrophys. J., 433, 229 (1994).

[3] S. Wanajo, T. Kajino, G. J. Mathews, and K. Otsuki, Astrophys. J., 554, 578 (2001).

[4] S. Wanajo, N. Itoh, Y. Ishimaru, S. Nozawa, and T. C. Beers, Astrophys. J., 577, 853 (2002).

[5] S. Wanajo, Astrophys. J., 650, L79 (2006).

[6] S. Wanajo, S. Goriely, M. Samyn, and N. Itoh, Y., Astrophys. J., 606, 1057 (2004).

[7] Y. -Z. Qian and S. E. Woosley, Astrophys. J., 471, 331 (1996).

[8] K. Kotake, S. Yamada, and K. Sato, Astrophys. J., 595, 304 (2003).

[9] R. Walder, A. Burrows, C. D. Ott, E. Livne,I. Lichtenstadt, and M. Jarrah, Astrophys. J., 626, 317 (2005).

[10] L. Scheck, K. Kifonidis, H. -Th. Janka, and E. Müeller, Astron. Astrophys., 457, 963 (2006).

[11] Y. Ishimaru and S. Wanajo, Astrophys. J., 511, L33 (1999).

[12] Y. Ishimaru, S. Wanajo, W. Aoki, and S. G. Ryan, Astrophys. J., 600, L47 (2004). 